\title{
Whole-genome analysis of multiple wood ant population pairs supports similar speciation histories, but different degrees of gene flow, across their European range
}

\author{
Beatriz Portinha ${ }^{1,2, \#}$, Amaury Avril ${ }^{3}$, Christian Bernasconi ${ }^{4}$, Heikki Helanterä ${ }^{5}$, Josie \\ Monaghan $^{6}$, Bernhard Seifert ${ }^{7}$, Vitor C. Sousa ${ }^{2, *}$, Jonna Kulmuni ${ }^{1,8, *}$, Pierre Nouhaud ${ }^{1, *, \#}$
}

\author{
Affiliations \\ 1: Organismal \& Evolutionary Biology Research Programme, University of Helsinki, Helsinki, Finland \\ 2: cE3c, Centre for Ecology, Evolution and Environmental changes, Faculdade de Ciências, Universidade \\ de Lisboa, Campo Grande, Edifício C2, 1749-016, Lisboa, Portugal \\ 3: Department of Ecology and Evolution, University of Lausanne, 1015 Lausanne, Switzerland \\ 4: Alpine Foundation for Life Science, $\mathrm{CH}-6718$ Blenio, Switzerland \\ 5: Ecology and Genetics research unit, University of Oulu, PO Box 3000, 90014 Oulu, Finland \\ 6: Department of Biology, University of York, York, UK \\ 7: Senckenberg Museum of Natural History Görlitz, Görlitz, Germany \\ 8: Tvärminne Zoological Station, University of Helsinki, Hanko, Finland \\ *: Co-last authors \\ \#: Corresponding authors: BP, bcportinha@gmail.com; PN, pierr3.nouhaud@gmail.com
}

\begin{abstract}
The application of demographic history modeling and inference to the study of divergence between species is becoming a cornerstone of speciation genomics. The demographic history is usually reconstructed by analysing a single population from each species, assuming that the divergence history inferred between these populations represents the actual speciation history. However, this assumption is rarely explicitly tested, and it may not be met when species diverge with gene flow. For instance, secondary contact between two species after a range expansion may be confined into a specific geographic region. In this study, we tested to what extent the divergence history inferred from two heterospecific populations would vary depending on their geographic locations, using mound-building red wood ants. The wood ant species Formica polyctena and F. aquilonia have contrasting distributions in Europe and naturally hybridize in Finland. We first performed whole-genome resequencing of 20 individuals sampled in multiple populations across both species ranges. We then reconstructed the divergence histories of distinct heterospecific population pairs using a coalescent-based approach. We found that the analysis of these different population pairs always supported a scenario of divergence with gene flow, suggesting that species divergence started in the Pleistocene (ca. $500 \mathrm{kya}$ ) and occurred with continuous asymmetrical gene flow from $F$. aquilonia to $F$. polyctena until a recent time, when migration stopped (2-19 kya, depending on the population pair considered). However, we found support for contemporary gene flow in the sympatric population pair from Finland, where hybrids have been described. Overall, our results suggest that divergence histories reconstructed from a few individuals may be reliable and applicable at the species level. Nonetheless, the geographical context of populations chosen to represent their species should be taken into account, as it may affect estimates of migration rates between species when gene flow is heterogeneous across their geographical ranges.
\end{abstract}




\section{INTRODUCTION}

Reconstructing divergence histories using genetic data has become gold standard in speciation genomics (Ravinet et al., 2017), which has been eased by the development of sequencing technologies and inference tools (Beichman et al., 2018). Classically, the divergence history between two related species is inferred using samples from a single pair of populations, one from each species, with the purpose of estimating key evolutionary parameters such as divergence times, migration rates, and effective population sizes (e.g., Nadachowska-Brzyska et al. 2013; Yagi et al., 2019; Sutra et al., 2019). Such an approach implicitly assumes that the divergence history between the two sampled populations is representative of the divergence history of the species as a whole, i.e. across their whole ranges. This assumption is expected to hold if species diverge in allopatry without gene flow. However, this assumption is rarely explicitly tested, and when divergence occurs with gene flow it is unclear to what extent parameter estimates may fluctuate across both species ranges.

Gene flow between two diverging lineages can vary through time (Sousa \& Hey 2013) and space. For instance, secondary contact between two species after a range expansion is likely to only affect populations in a specific geographic region (e.g., Green et al., 2010). In such cases, reconstructing the divergence history between the two species would depend on the geographic location of the set of populations sampled, because populations also evolve in space (see Bradburd \& Ralph, 2019 for a recent review on spatial population genetics). While some studies have previously reconstructed the divergence history between several species using multiple population pairs (e.g., Zieliński et al., 2016; Filatov et al., 2016; Pabijan et al., 2017; Rougemont and Bernatchez, 2018; Ito et al., 2020; Garcia-Erill et al., 2021), to our knowledge variation inferred between the multiple comparisons has not been reported. In this study, we investigate how the divergence history between two species may vary depending on the geographic location of the pair of heterospecific populations considered, using mound-building red wood ants.

Red wood ants of the Formica rufa group (Hymenoptera, Formicidae) play important ecosystemic roles in boreal forests (Frouz et al. 2016; Stockan et al. 2016) and are a good system to study variability in the divergence history across species geographical 
ranges. This is because the Palearctic F. rufa group encompasses up to 13 species (Seifert 2021), many with different distribution areas and that likely experienced gene flow or secondary contact in different regions. Phylogenetic studies using mitochondrial markers suggest that speciation within this group took place in the Pleistocene, in the last 500,000 years (Goropashnaya et al., 2004; Goropashnaya et al., 2012). While their speciation history is unknown, they may have diverged in different forest refugia during Pleistocene glaciations (Goropashnaya et al., 2004). Among the F. rufa group, $F$. polyctena and $F$. aquilonia are two non-sister species with contrasting distributions. Within the European part of their Palaearctic ranges, F. aquilonia shows a borealsubarctic horizontal and highly montane-subalpine vertical distribution, whereas $F$. polyctena is largely temperate-subboreal and planar to submontane (Seifert, 2018). Both species overlap in Switzerland (where they occupy different altitudinal niches) and around the Baltic sea, and natural hybrids have been characterized in Southern Finland (Kulmuni et al., 2010; Beresford et al., 2017).

Here, we reconstructed the speciation history between $F$. polyctena and $F$. aquilonia using whole-genome data by contrasting multiple pairs of populations sampled from both species across Europe. Our aim was to understand to what extent different population pairs yield similar demographic histories and parameter estimates. The chosen pairs of populations represent situations of present-day strict allopatry (West Switzerland F. polyctena vs. Scotland F. aquilonia and East Switzerland F. polyctena vs. Scotland F. aquilonia), allopatry/parapatry in Switzerland (where species are distributed over different altitudinal ranges, but opportunities for gene flow cannot be ruled out completely; Cherix et al., 2012) and sympatry in Finland (where hybridization has been characterized). Our results suggest that divergence started in the Pleistocene and that it occured with continuous asymmetric gene flow from $F$. aquilonia to $F$. polyctena. Interestingly, all population comparisons supported the same divergence scenario, with comparable divergence times ranging from 523,900 to 561,745 years ago, in agreement with previous findings. Nevertheless, we also found differences between population pairs, particularly in contemporary gene flow estimates. Among these differences, we found support for increased bidirectional migration at recent times only in Finland, where gene flow could be mediated by hybrids. By using multiple populations distributed across both species ranges, we were 
able to both draw a consistent picture of the speciation history (i.e., divergence times, ancestral effective sizes and ancestral migration rates) while uncovering variability in migration rates which could be explained by local opportunities for gene flow.

\section{MATERIALS AND METHODS}

\section{Study system}

Formica polyctena and F. aquilonia are two Holarctic ant species of the Formica genus that inhabit coniferous and mixed forests. They are haplodiploid (females are diploid and males are haploid) and arrhenotokous (mothers produce male offspring from unfertilized eggs; De La Filia et al., 2015). They are social insects and, as such, labour is divided between reproductive queens and workers. As both of these species are polygynous, each nest may have hundreds of egg-laying queens of different ages. The species are also supercolonial, meaning that populations are formed by the association of many cooperating nests (hereafter, population is used as a synonym for supercolony). Polygyny results in low relatedness among individuals sampled within the same nest and/or supercolony (e.g., Sundström et al., 2005). New queens are produced in the Spring and they may shed their wings without a nuptial flight. As such, matings can happen with males from their own population. Finally, if long-range dispersal occurs, it is likely male-biased (Maeder et al., 2016).

\section{Sampling}

Our main aim was to understand to what extent samples from different geographical locations yield similar demographic histories and parameter estimates. To address this, females (workers) of each parental species were sampled from several locations across Europe (Fig. 1). For Formica polyctena, sampling was carried out in two locations in Switzerland (East and West) and in the Åland islands (Finland). For each sampling site, three female workers were sampled from different populations, and/or different nests within the same population, whenever possible (Table 1). In addition, one more female of each species was collected in Southern Finland, where hybridization between both species has been previously documented (Beresford et al., 
2017). Overall, ten females were sampled for each species (20 individuals in total, Table 1).

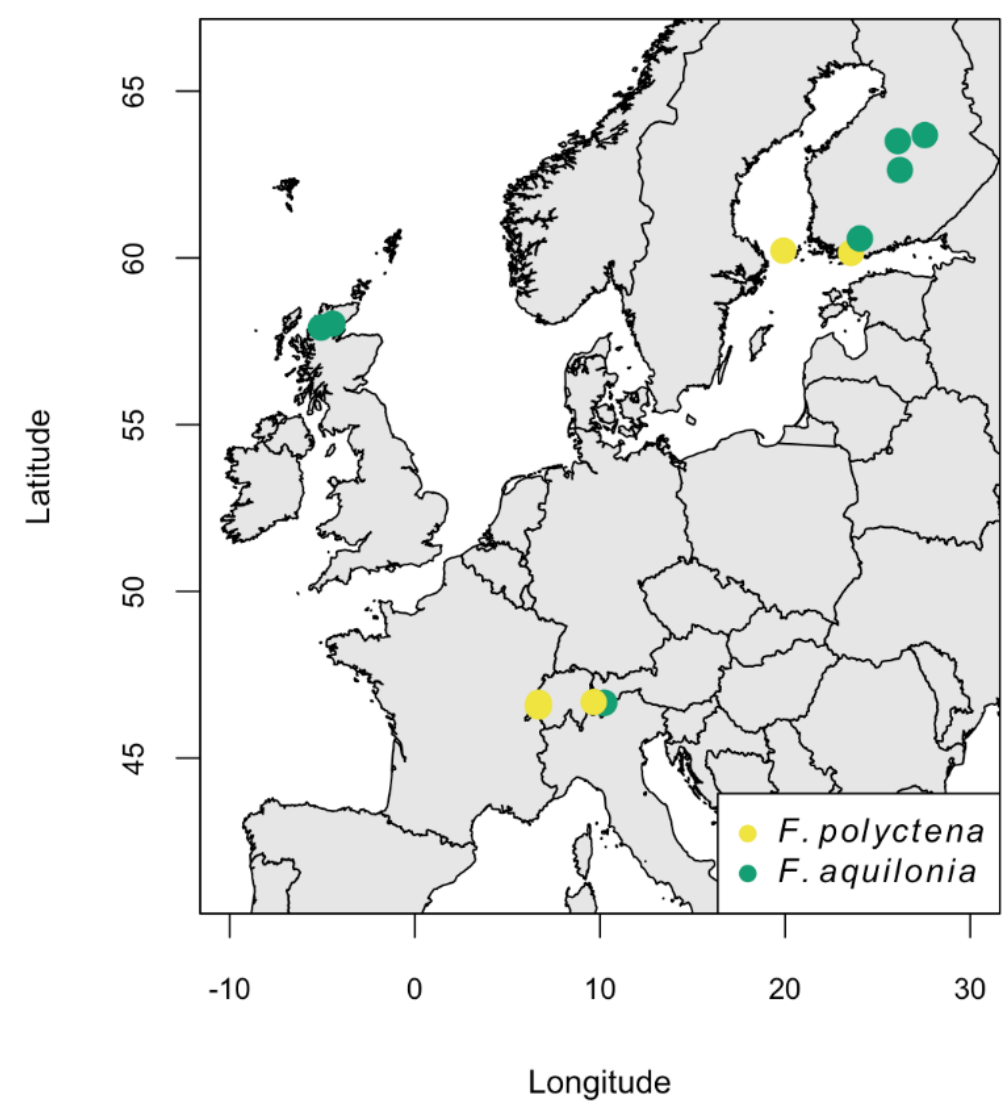

Figure 1 - Map of sampling locations. Each symbol represents a sampled individual (some are overlapping).

\section{Morphological identification}

Prior to sequencing, a subset of samples (at least one nest per geographical location, Table 1) were morphologically identified at the species level using Numeric Morphology-Based Alpha-Taxonomy (NUMOBAT). Overall, 16 morphological characters were investigated (CS, CL/CW, SL/CS, nCH, OccHL, nGU, GUHL, nPN, $\mathrm{mPnHL}, \mathrm{nMes}$, nMet, MetHL, nPr, EyeHL, nSc and SL/Smax, Seifert, 2018).

\section{DNA extraction and sequencing}

For all samples, DNA was extracted from whole-bodies with a SDS (sodium dodecyl sulfate) protocol. DNA libraries were constructed using NEBNext DNA Library Prep 
Kits (New England Biolabs). Samples were processed and sequenced at Novogene (Hong Kong) as part of the Global Ant Genomics Alliance (Boomsma et al., 2017). Whole-genome sequencing was performed on Illumina Novaseq 6000 (150 base pairs paired-end reads), targeting $15 \times$ per individual.

Raw Illumina reads and adapter sequences were trimmed using Trimmomatic (v0.38; parameters LEADING:3, TRAILING:3, MINLEN:36; Bolger et al., 2014) before mapping against the reference genome (Nouhaud et al., 2021) using BWA MEM with default parameters (v0.7.17; Li and Durbin, 2010). Duplicates were removed using Picard Tools with default parameters (v2.21.4; http://broadinstitute.github.io/picard).

Table 1 - Sample information. Sampling location (locality, geographic coordinates and altitude), nest of origin, ancestry proportions (reconstructed by the sNMF analysis for $\mathrm{K}=2$ ), and assignment probabilities for the 20 sampled individuals.

\begin{tabular}{|c|c|c|c|c|c|c|c|c|c|c|c|}
\hline \multirow{2}{*}{ Species } & \multirow{2}{*}{$\begin{array}{l}\text { Geographical } \\
\text { location }\end{array}$} & \multirow{2}{*}{ Population } & \multirow{2}{*}{ Nest } & \multirow{2}{*}{ Sample ID } & \multirow{2}{*}{ Latitude } & \multirow{2}{*}{ Longitude } & \multirow{2}{*}{$\begin{array}{l}\text { Altitude } \\
\text { (meters) }\end{array}$} & \multicolumn{2}{|c|}{$\begin{array}{c}\text { Admixture } \\
\text { proportions (\%) }\end{array}$} & \multicolumn{2}{|c|}{ Morphological identification } \\
\hline & & & & & & & & Cluster 1 & Cluster 2 & NUMOBAT & Probability \\
\hline \multirow{9}{*}{ 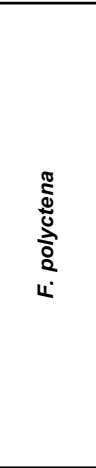 } & $\begin{array}{l}\text { Finland } \\
\text { Finland }\end{array}$ & $\begin{array}{c}\text { Attbole } \\
\text { Jarso }\end{array}$ & $\begin{array}{l}\text { Att1 } \\
\text { Jar6 }\end{array}$ & $\begin{array}{l}\text { Att1_1w } \\
\text { Jar6_1w }\end{array}$ & $\begin{array}{l}60.215 \\
60.014\end{array}$ & $\begin{array}{l}19.907 \\
20.001\end{array}$ & $\begin{array}{l}27 \\
13\end{array}$ & $\begin{array}{l}71.98 \\
71.20\end{array}$ & $\begin{array}{l}28.02 \\
28.80\end{array}$ & - & - \\
\hline & Finland & Lokholm & Lok3 & Lok3_1w & 60.375 & 19.810 & 5 & 69.16 & 30.84 & F. polyctena & 0.729 \\
\hline & Finland & Fiskars & Fis2 & Fis2_1w & 60.151 & 23.557 & 66 & 81.24 & 18.76 & $\begin{array}{l}F \text {. rufa } \times F \text {. } \\
\text { polyctena }\end{array}$ & 0.991 \\
\hline & $\begin{array}{l}\text { Western } \\
\text { Switzerland }\end{array}$ & $\begin{array}{c}\text { Chalet a } \\
\text { Gobet }\end{array}$ & $\mathrm{CAGa}$ & CAGa_1w & 46.546 & 6.688 & 813 & 99.99 & 0.01 & F. polyctena & 0.999 \\
\hline & $\begin{array}{l}\text { Western } \\
\text { Switzerland }\end{array}$ & $\mathrm{Naz}$ & $\mathrm{NAZa}$ & NAZa_1w & 46.660 & 6.684 & 680 & 99.99 & 0.01 & - & - \\
\hline & $\begin{array}{l}\text { Western } \\
\text { Switzerland }\end{array}$ & $\begin{array}{l}\text { Vernand } \\
\text { Dessus }\end{array}$ & VDa & VDa_1w & 46.577 & 6.628 & 690 & 96.26 & 3.74 & - & - \\
\hline & $\begin{array}{c}\text { Eastern } \\
\text { Switzerland }\end{array}$ & Alvaneu & $\mathrm{CBCH} 1$ & $\mathrm{CBCH} 1 \_1 w$ & 46.681 & 9.657 & 1236 & 99.99 & 0.01 & - & - \\
\hline & $\begin{array}{c}\text { Eastern } \\
\text { Switzerland }\end{array}$ & Alvaneu & $\mathrm{CBCH} 2$ & $\mathrm{CBCH} 2$ 2w & 46.681 & 9.657 & 1236 & 99.99 & 0.01 & - & - \\
\hline & $\begin{array}{c}\text { Eastern } \\
\text { Switzerland }\end{array}$ & Alvaneu & $\mathrm{CBCH} 3$ & $\mathrm{CBCH} 3 \_1 w$ & 46.681 & 9.657 & 1236 & 99.99 & 0.01 & F. polyctena & 0.989 \\
\hline \multirow{10}{*}{ 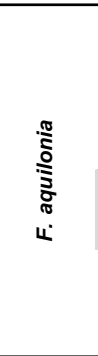 } & $\begin{array}{c}\text { Eastern } \\
\text { Switzerland }\end{array}$ & Stabelchod & CBAQ1 & CBAQ1_1w & 46.661 & 10.230 & 1881 & 0.01 & 99.99 & F. aquilonia & 0.9995 \\
\hline & $\begin{array}{c}\text { Eastern } \\
\text { Switzerland }\end{array}$ & Stabelchod & CBAQ3 & CBAQ3_1w & 46.661 & 10.230 & 1881 & 6.17 & 93.83 & - & - \\
\hline & $\begin{array}{c}\text { Eastern } \\
\text { Switzerland }\end{array}$ & $\begin{array}{l}\text { Alp La } \\
\text { Schera }\end{array}$ & CBAQ2 & CBAQ2_2w & 46.653 & 10.189 & 1716 & 0.97 & 99.03 & - & - \\
\hline & Scotland & Lairg & Lai & Lai_1w & 58.028 & -4.441 & 108 & 0.01 & 99.99 & F. aquilonia & 0.9809 \\
\hline & Scotland & Lairg & Lai & Lai_2w & 58.028 & -4.441 & 108 & 0.01 & 99.99 & - & - \\
\hline & Scotland & Loch Achall & Loa & Loa_1w & 57.910 & -5.082 & 77 & 0.01 & 99.99 & - & - \\
\hline & Finland & Pukara & CF14a & CF14a_1w & 62.635 & 26.201 & 124 & 3.02 & 96.98 & - & - \\
\hline & Finland & Koivula & CF4b & CF4b_1w & 63.503 & 26.087 & 150 & 2.27 & 97.73 & F. aquilonia & 0.993 \\
\hline & Finland & Sonkajarvi & CF8b & CF8b_1w & 63.682 & 27.545 & 117 & 4.42 & 95.58 & - & - \\
\hline & Finland & Pusula & Pus2 & Pus2 1w & 60.581 & 24.038 & 129 & 0.01 & 99.99 & F. aquilonia & 0.984 \\
\hline
\end{tabular}

\section{SNP calling and filtering}

Single nucleotide polymorphisms (SNPs) and genotypes were called across all samples with freebayes (v1.3.1; Garrison and Marth, 2012), disabling population priors 
(-k). After SNP calling, the VCF was normalised using vt (v0.5; Tan et al., 2015) and sites located at less than two base pairs from indels were excluded, along with sites supported by only Forward or Reverse reads. Multi-nucleotide variants were decomposed with the vcfallelicprimitives command from vcflib (v1.0.1).

Only biallelic SNPs with quality equal or higher than 30 were kept. In order to refrain from removing entire sites when only a subset of individuals had inadequate genotype calls, individual genotypes with genotype qualities lower than 30 were coded as missing data. Genotypes with depth of coverage lower than eight were also coded as missing data, after which sites with missing data across more than half of our samples were removed.

To remove genotyping errors that cause sites to show excessive heterozygosity (e.g., due to unresolved paralogs or alignment errors), we first pooled all samples together, creating a Wahlund effect on purpose, after which we applied a filter based on HardyWeinberg Equilibrium and excluded sites displaying heterozygote excess $(p<0.01$; see e.g., Pfeifer et al., 2018).

We applied a filter based on sequencing depth by setting individual-specific thresholds: sites were only kept if their coverage was between half and twice the mean individual value. Finally, we removed sites located on the third chromosome, also known as the social chromosome. This chromosome harbours genes responsible for polymorphism in social organization in Formica species, controlling if a colony is headed by one (monogynous) or multiple (polygynous) queens (Brelsford et al., 2020). Recombination is rare between monogynous and polygynous alleles of this chromosome (supergene, Brelsford et al., 2020), leading to the maintenance of ancestral polymorphisms across Formica species which could bias our demographic inference.

\section{Population structure}

Population structure was studied by means of two individual-based methods, Principal Component Analysis (PCA) and sNMF clustering analysis (Frichot et al., 2014), the latter of which estimates individual ancestry coefficients. These analyses were performed in R (v3.6.3; R Core Team, 2017) using the LEA package (v3.0.0; Frichot 
and François, 2015). The sNMF analysis was repeated 20 times for a number of ancestral clusters $(\mathrm{K})$ ranging from 2 to 8 . The $\mathrm{K}$ value with lowest cross-entropy obtained by sNMF was considered as the best $\mathrm{K}$ value, and the run with the lowest cross-entropy for the best $\mathrm{K}$ value was considered as the best run.

Observed and expected heterozygosity, inbreeding coefficients ( $\left.F_{I S}\right)$ and pairwise fixation indices ( $F_{\mathrm{ST}}$; Weir and Cockerham, 1984) were calculated using custom scripts. Pairwise $F_{\mathrm{ST}}$ values were also computed between populations using the SNPRelate package (v1.20.1; Zheng et al., 2012).

A

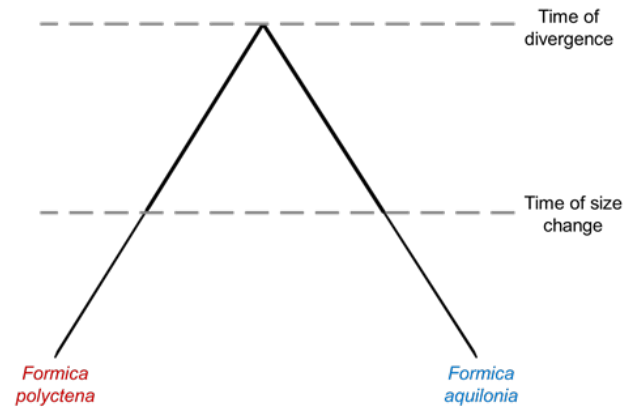

C

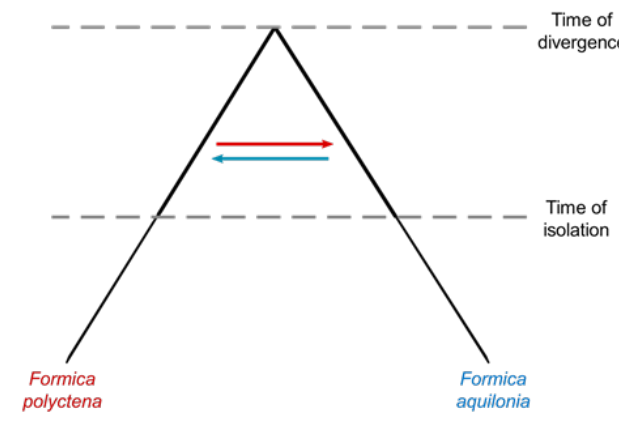

B

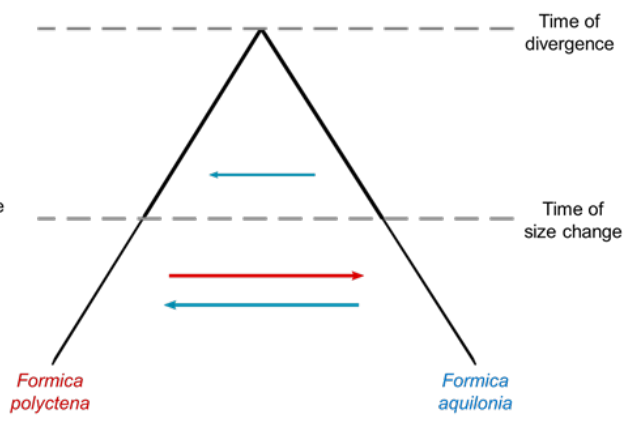

D

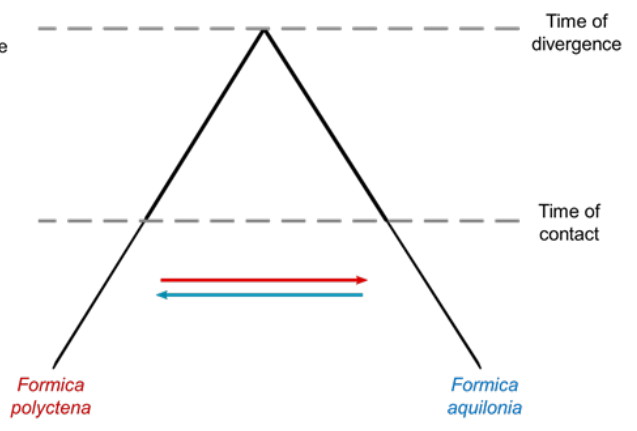

Figure 2 - Demographic models designed to study the speciation history between Formica polyctena and $F$. aquilonia. A Allopatry scenario. B Sympatry scenario, allowing for different migration rates throughout the divergence of the populations. C Isolation after migration scenario. D Migration after isolation scenario. Arrows represent migration. The direction of gene flow is indicated by the direction and colour of the arrows (red represents gene flow from $F$. polyctena into $F$. aquilonia; blue represents gene flow from $F$. aquilonia into $F$. polyctena). The different thickness in the lines representing the populations represent changes in effective size, which can happen either by instantaneous contractions or expansions. 


\section{Demographic modelling}

To document the divergence history across the species ranges, we compared alternative demographic models to demographic parameters inferred from the site frequency spectrum (SFS) obtained from different combinations of populations. This was done using the composite-likelihood method implemented in fastsimcoal2 (v2.6; Excoffier et al., 2013, parameters detailed in Supplementary Table 1). For allopatric cases, we considered three population pairs: $F$. polyctena from West Switzerland vs. F. aquilonia from Scotland; F. polyctena from East Switzerland vs. F. aquilonia from Scotland and F. polyctena from West Switzerland vs. F. aquilonia from East Switzerland. The sympatric population pair was formed by Finnish populations of both species. Each model was run 100 times, with 80 iterations per run for likelihood maximization, and 200,000 coalescent simulations per iteration to approximate the expected SFS. The mutation rate was assumed as $3.5 \times 10^{-9}$ per bp per haploid genome per generation, which is approximated from estimates available for social insects (Liu et al., 2017). No population growth was assumed (i.e., population growth rates were zero).

In the Formica genus, young queens can start laying eggs in their first years of life and have been estimated to live up to five years for F. polyctena (Horstmann, 1983), with queens of different ages within a single nest (i.e., overlapping generations). As such, generation time was assumed to be 2.5 years.

After obtaining point parameter estimates and expected likelihoods for all models (see below) tested with all population comparisons considered, the model with the highest expected likelihood was chosen as the best model in each case.

\section{Speciation history between $F$. polyctena and $F$. aquilonia}

To ascertain whether the speciation history between $F$. aquilonia and $F$. polyctena is different across both species ranges, we considered four divergence scenarios: allopatry, sympatry, isolation after migration, and migration after isolation (Fig. 2). The first two models allow for populations to change sizes at any time (Fig. 2A,B), while the remaining models allow resize events only when migration changes (Fig. 2C,D). The 
model with a scenario of sympatry between the populations (Fig. 2B) allows the migration rates between populations to change after the population sizes change. Parameter search ranges were improved for this particular model based on preliminary analyses, in order to decrease the probability that the parameter estimates obtained represent local maxima.

\section{Ghost introgression}

In order to rule out the possibility that the signal of gene flow we detect between $F$. aquilonia and $F$. polyctena is actually caused by gene flow from an unsampled (socalled ghost) species into either of the focal species, we modeled two different ghost scenarios which are based on species relationships within the $F$. rufa group as described in Goropashnaya et al. (2012). The first scenario models ghost introgression from $F$. rufa, which is a sister species to $F$. polyctena, and which may send migrants to either F. polyctena (Fig. 3A), or F. aquilonia (Fig. 3C). The second, alternative scenario models ghost introgression from either F. lugubris and/or F. paralugubris, which are in the same clade as $F$. aquilonia, and which may send migrants to either $F$. aquilonia (Fig. 3B), or F. polyctena (Fig. 3D). These models purposefully did not consider direct migration between $F$. polyctena and $F$. aquilonia, since they were designed to rule out the possibility that the signal of gene flow between the focal species could be caused by gene flow from an unsampled sister species.

\section{SFS characteristics}

To perform the demographic analyses detailed above, we built SFSs using data from two populations (2D-SFS). As we lack a good outgroup to infer the ancestral state of the alleles in our dataset, folded SFSs were built using the minor allele frequency (MAF) method. We ensured that there was no missing data by downsampling genotypes. To do this, a minimum sample size across all sites was determined (corresponding to the number of individuals to resample from minus the maximum number of missing data per site). Individuals were resampled in windows of $50 \mathrm{Kbp}$, discarding blocks where the mean distance between consecutive SNPs in a given 
block was less than $2 \mathrm{bp}$. To maximize the number of sites that could be kept, the individuals selected to be resampled in each window were the ones with higher amounts of data in that specific window.

All SFSs included the number of monomorphic sites, which, in conjunction with a mutation rate, allows scaling parameter estimates inferred by the models (e.g. to obtain divergence times in number of generations). We estimated these numbers using the proportion of polymorphic sites in relation to the total number of callable sites of individuals in a specific dataset. The total number of callable sites was obtained for each individual using mosdepth (v0.2.9; Pedersen \& Quinlan, 2017) considering individual depth of coverage thresholds defined earlier for SNP calling.
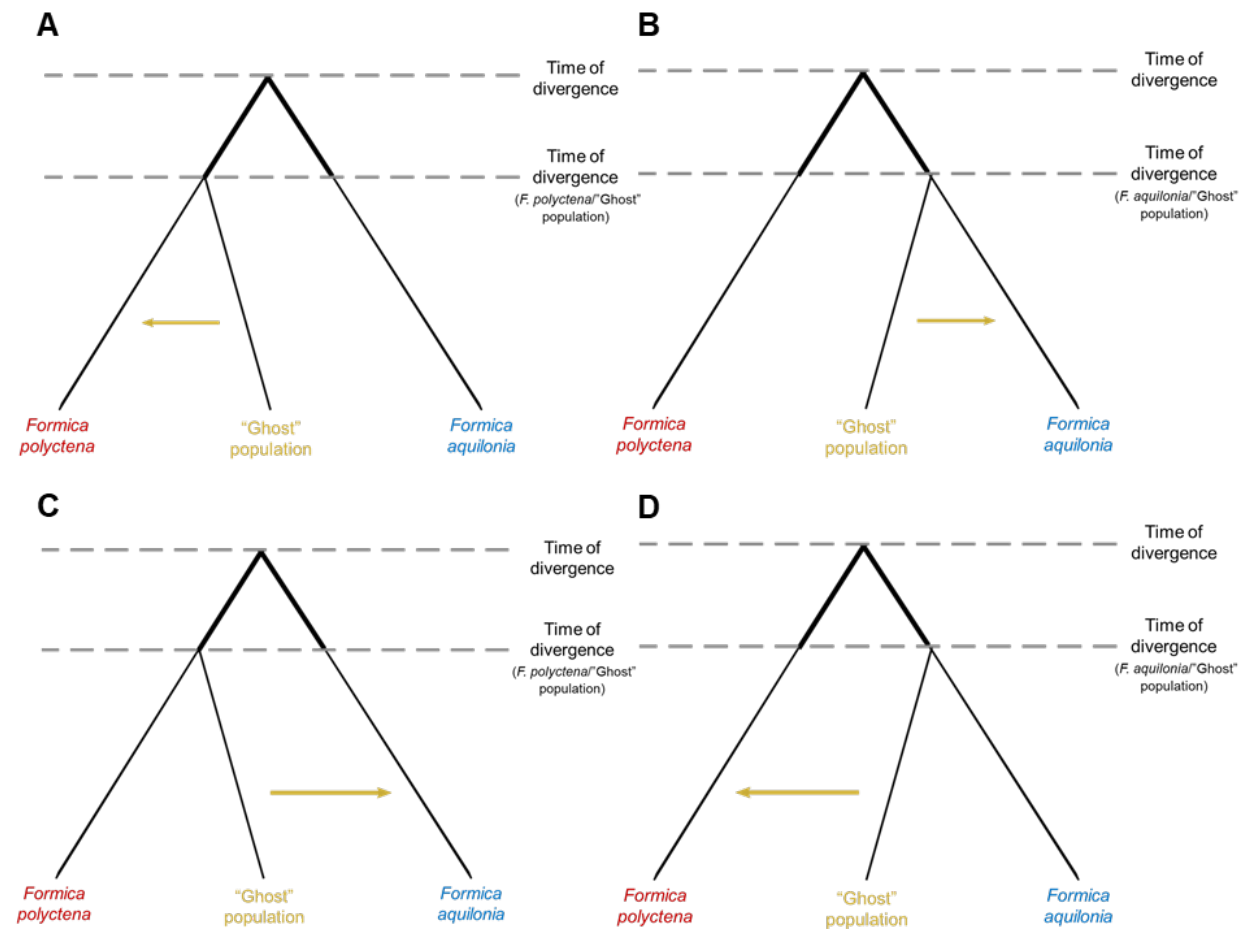

Figure 3 - Demographic models designed to study possible introgression from unsampled species ("ghost") into Formica polyctena or F. aquilonia. A Scenario where the unsampled species is divergent from $F$. polyctena, into which it sends migrants. B Scenario where the unsampled species is divergent from $F$. aquilonia, into which it sends migrants. C Scenario where the unsampled species is a sister of $F$. polyctena and sends migrants into $F$. aquilonia. D Scenario where the unsampled species is a sister of $F$. aquilonia and send migrants into $F$. polyctena. Gene flow and changes in population sizes are depicted as in Figure 2. 


\section{RESULTS}

\section{Sequencing and SNP calling}

Illumina sequencing yielded on average $7.12 \mathrm{~Gb}$ of raw data per sample (min: 5.87, max: 8.29). After trimming, mapping and filtering, average sequencing depth was $15.6 \times$ \pm 1.66 (standard deviation). SNP calling using Freebayes recovered 2,856,374 biallelic sites with quality values above 30 . Among these sites, 2,211,441 were left after filtering on sequencing depth, individual coverage and excessive heterozygosity, and $2,054,352$ after removing sites located on the third chromosome. The fraction of missing data per site averaged $15.49 \%$ across all 20 individuals in the final SNP set.

\section{Morphological species identification}

A subset of samples used for genomic analyses were also used in morphological species identification. The analysis of 16 morphological characters under the NUMOBAT framework supported our prior species assignment for all $F$. aquilonia samples and non-Finnish F. polyctena samples. For these samples, all posterior probabilities of the morphological assignment were greater than $98 \%$ (Table 1 ). Individual samples collected in Åland (Lokholm) were morphologically assigned as $F$. polyctena $(72.9 \%)$ and $F$. polyctena $\times$ F. aquilonia hybrids $(26.8 \%)$, while samples collected in Fiskars were assigned as F. polyctena $\times$ F. rufa hybrids (99.1\%, Table 1). The clustering analysis based on the genome-wide data carried with sNMF assigned these two individuals (Lok3_1w and Fis2_1w, respectively; Table 1) as admixed F. polyctena (69.16 and $81.24 \%$ assignment proportions, respectively; see below and Table 1). As such they were included in all analyses with the prior that they may be admixed themselves.

\section{Summary statistics and genetic structure}

Genome-wide, average pairwise differentiation indices $\left(F_{\mathrm{ST}}\right)$ for all possible combinations of geographic sampling locations were moderately high $\left(F_{\mathrm{ST}}>0.1\right)$ in all cases (Table 2). The highest value was recorded between $F$. polyctena individuals 
from East Switzerland and $F$. aquilonia individuals from Scotland $\left(F_{\mathrm{ST}}=0.497\right)$, and the lowest between the $F$. polyctena individuals from Finland and West Switzerland $\left(F_{\mathrm{ST}}=0.113\right)$. Average differentiation between intraspecific samples of the parental species was 0.202 for $F$. aquilonia and 0.131 for $F$. polyctena. Interspecific differentiation ranged from 0.256 to 0.497 . Average interspecific differentiation was 0.398 .

Table 2 - Pairwise fixation indexes $\left(F_{\mathrm{ST}}\right)$ between geographic sampling locations of Formica polyctena and $F$. aquilonia used in this study.

\begin{tabular}{|c|c|c|c|c|c|c|c|}
\hline & & \multicolumn{3}{|c|}{ F. polyctena } & \multicolumn{3}{|c|}{ F. aquilonia } \\
\hline & & Finland & $\begin{array}{c}\text { West } \\
\text { Switzerland }\end{array}$ & $\begin{array}{c}\text { East } \\
\text { Switzerland }\end{array}$ & Switzerland & Scotland & Finland \\
\hline \multirow{3}{*}{$\begin{array}{l}\frac{0}{2} \\
\frac{d}{0} \\
\frac{2}{0} \\
\frac{2}{2} \\
4\end{array}$} & Finland & - & 0.113 & 0.160 & 0.283 & 0.300 & 0.256 \\
\hline & $\begin{array}{c}\text { West } \\
\text { Switzerland }\end{array}$ & - & - & 0.120 & 0.444 & 0.462 & 0.413 \\
\hline & $\begin{array}{c}\text { East } \\
\text { Switzerland }\end{array}$ & - & - & - & 0.480 & 0.497 & 0.445 \\
\hline \multirow{3}{*}{ 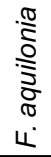 } & Switzerland & - & - & - & - & 0.213 & 0.189 \\
\hline & Scotland & - & - & - & - & - & 0.204 \\
\hline & Finland & - & - & - & - & - & - \\
\hline
\end{tabular}

PCA clearly separated the parental species into two ends of the first principal component (PC, Fig 4). PC1 explained $\sim 29 \%$ of the variation in the data and was statistically significant $(p<0.01$; Supplementary Figure 1). Finnish individuals of $F$. polyctena were plotted closer to $F$. aquilonia individuals, when compared to other nonFinnish F. polyctena individuals.

Table 3 - Mean expected $\left(\mathrm{H}_{\mathrm{e}}\right)$ and observed $\left(\mathrm{H}_{\mathrm{o}}\right)$ heterozygosities and mean inbreeding coefficient (FIS) of the geographic sampling locations.

\begin{tabular}{lcccc}
\hline Species & Sampling location & $\boldsymbol{H}_{\mathrm{e}}$ & $\boldsymbol{H}_{\mathrm{o}}$ & $\boldsymbol{F}_{\mathrm{IS}}$ \\
\hline \multirow{3}{*}{ F. polyctena } & Finland & 0.185 & 0.169 & 0.087 \\
& West Switzerland & 0.134 & 0.123 & 0.082 \\
& East Switzerland & 0.120 & 0.123 & -0.029 \\
F. aquilonia & Switzerland & 0.131 & 0.114 & 0.129 \\
& Scotland & 0.123 & 0.103 & 0.165 \\
& Finland & 0.143 & 0.134 & 0.060 \\
\hline
\end{tabular}


The sNMF analysis considered two to eight possible ancestral clusters (K). Cross entropy analysis (Supplementary Figure 2) revealed that the best $\mathrm{K}$ value for our data was two (Table 1, Supplementary Figure 3). In this case, individuals of both parental species clustered with each other. Interestingly, Finnish individuals of each parental species show some ancestry from the opposite ancestral cluster, up to an ancestry proportion of $\sim 31 \%$ from the F. aquilonia ancestral cluster in Finnish F. polyctena and $\sim 4.42 \%$ from the F. polyctena ancestral cluster in Finnish F. aquilonia. Overall, the sNMF analysis agreed with the morphological identification carried with 16 characters.

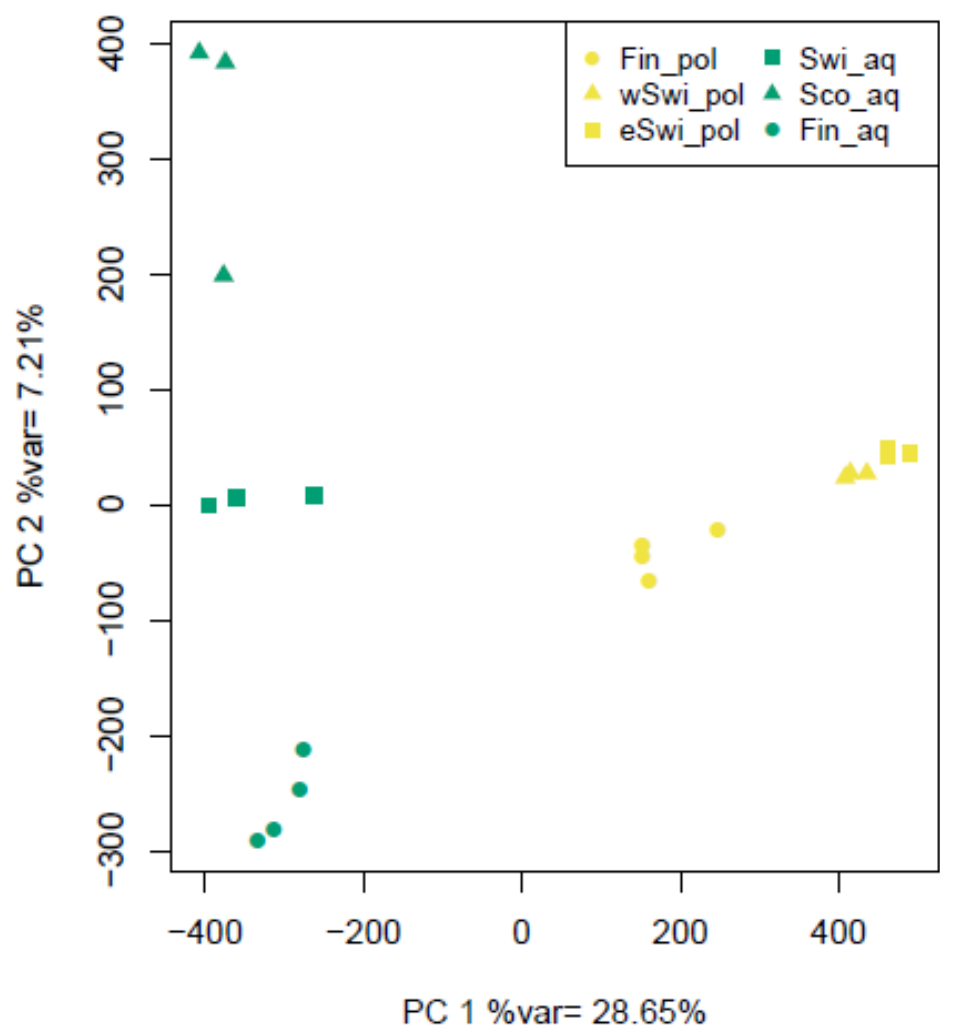

Figure 4 - Principal Component Analysis. Principal Component (PC) 1 is shown plotted against PC2. Formica polyctena individuals are shown in yellow, with $F$. aquilonia females shown in green. Each point represents an individual and the different point symbols represent the different geographic sampling locations. Abbreviations are as follows: Fin_pol $=F$. polyctena in Finland; wSwi_pol $=F$. polyctena in West Switzerland; eSwi_pol $=F$. polyctena in East Switzerland; Swi_aq $=F$. aquilonia in Switzerland; Sco_aq $=F$. aquilonia in Scotland; Fin_aq $=$ F. aquilonia in Switzerland

Mean expected heterozygosity $\left(H_{\mathrm{e}}\right)$ per sampling location ranged from 0.120 to 0.185 (Table 3). F. aquilonia from Scotland had the lowest value (0.120), while F. polyctena from Finland had the highest $H_{\mathrm{e}}(0.185)$. Mean observed heterozygosity $\left(H_{\mathrm{o}}\right.$; Table 3$)$ 
per location ranged from 0.103 (Scotland F. aquilonia) to 0.169 (Finland F. polyctena). All populations, excluding F. polyctena from East Switzerland, had lower observed than expected heterozygosity values, translating to positive inbreeding coefficients ( $F_{\mathrm{IS}}$, Table 3). The heterozygosity values are not strictly comparable across locations, since some locations have all samples coming from different populations, whereas other locations consist of samples from the same population (Table 1).

\section{All comparisons support a scenario of divergence with gene flow}

In order to study the speciation history between F. polyctena and F. aquilonia, we first analysed locations where no hybridization had been previously detected, focusing on the following pairs: (i) F. polyctena from Western Switzerland vs. F. aquilonia from Scotland (allopatric), (ii) F. polyctena from Eastern Switzerland vs. F. aquilonia from Scotland (allopatric) and (iii) F. polyctena from Western Switzerland vs. F. aquilonia from Eastern Switzerland (allopatric/parapatric). For all these population comparisons, the model that implemented divergence in sympatry was found to be the best fit (Fig. 6; Supplementary Tables 2-4 for parameter estimates obtained with all models). The direction of gene flow, migration rates, divergence times and ancestral population sizes were consistent between comparisons involving different pairs of locations (Fig. 5, panels $A, B$ and $C$ ).

First, gene flow between species was inferred to be asymmetrical, with migrants moving exclusively from $F$. aquilonia into $F$. polyctena at a rate averaging 0.5 migrants per generation $(2 \mathrm{Nm})$. Second, the time at which the populations of each species diverged was consistent across the different comparisons and ranged between 209,560 generations (Eastern Switzerland F. polyctena vs. Scotland F. aquilonia) and 213,277 generations (Western Switzerland F. polyctena vs. Scotland F. aquilonia). Assuming a generation time of 2.5 years, the estimates for the divergence between these species ranged from 523,900 to 533,193 years ago. Third, the effective size of the ancestral population of both species was consistently estimated to be between 460,000 and 500,000 haploid individuals across comparisons. After divergence, $F$. aquilonia was consistently inferred to have a larger $N_{e}$ than $F$. polyctena throughout their history. Finally, our results for all comparisons indicate that both species undergo 
simultaneous population size contractions which would have occurred between 801 (Western Switzerland F. polyctena vs. Scotland F. aquilonia) and 6,540 generations ago (Western Switzerland F. polyctena vs. Eastern Switzerland F. aquilonia). In years, these estimates place the times of population decline between 2,003 and 16,350 years ago.

\section{Sympatric Finnish samples support a similar speciation history, but with recent bidirectional gene flow}

As $F$. aquilonia and F. polyctena hybridize in Finland, we tested whether this could have an impact on the divergence history inferred when analyzing a pair of populations from this area. While the expected likelihood of the migration after isolation model was the highest for this population comparison (Supplementary Table 5 for parameter estimates obtained with all models), it was only 3.65 log units better than the likelihood of the model that considers divergence in sympatry. This suggests that both models fit this dataset equally well. As the parameter estimates (e.g., times of divergence and size change, ancestral population sizes) obtained under the sympatry scenario are comparable to those obtained with the comparisons outside of Finland, we consider that the sympatry model is a more parsimonious description of the demographic history between the Finnish F. polyctena and F. aquilonia individuals (Fig. 6D). The time of divergence estimated under the sympatry scenario for the Finnish comparison, 224,698 generations (561,745 years), was in line with previous estimates. The size of the ancestral population of both species in the model was also comparable to the estimates obtained for the other comparisons (431,527 haploid individuals), and the ancestral population sizes followed the previous trends, with larger estimates for $F$. aquilonia $(309,413$ haploids) than $F$. polyctena $(208,665)$. However, the size of the Finnish $F$. polyctena population increased in more recent times, while Finnish $F$. aquilonia still contracted, similar to non-Finnish $F$. aquilonia. The time of size change was estimated at 7,520 generations (18,800 years), slightly older than what was inferred for other comparisons. As observed for non-Finnish samples, prior to the population resizes there was unidirectional gene flow from $F$. aquilonia into $F$. polyctena in Finland. However, the migration rate was inferred at 1.23 migrants per 
generation, which was greater than the average $2 \mathrm{Nm}=0.5$ inferred from non-Finnish comparisons (see above). Moreover, in recent times, bidirectional gene flow was inferred, with 3.18 haploid migrants per generation moving from $F$. aquilonia into $F$. polyctena and 0.21 haploid individuals migrating in the other direction.

A

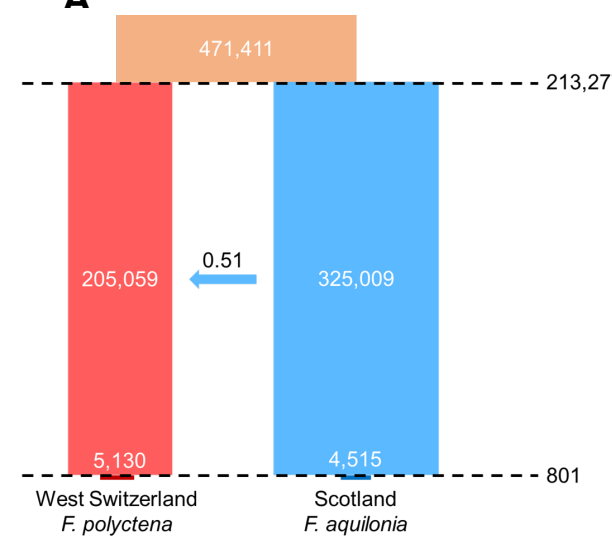

C

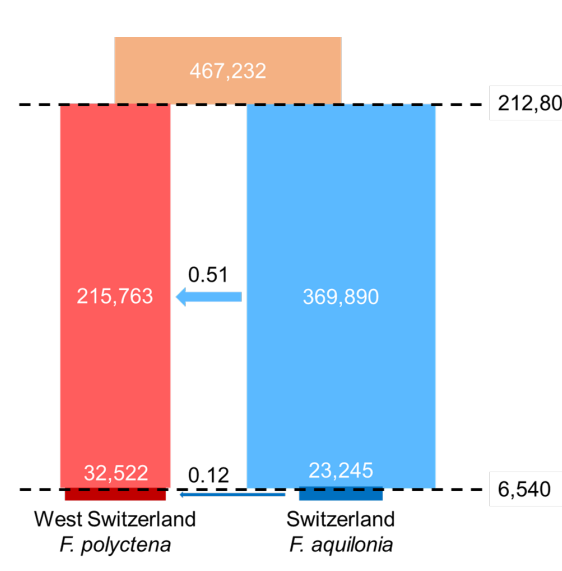

B

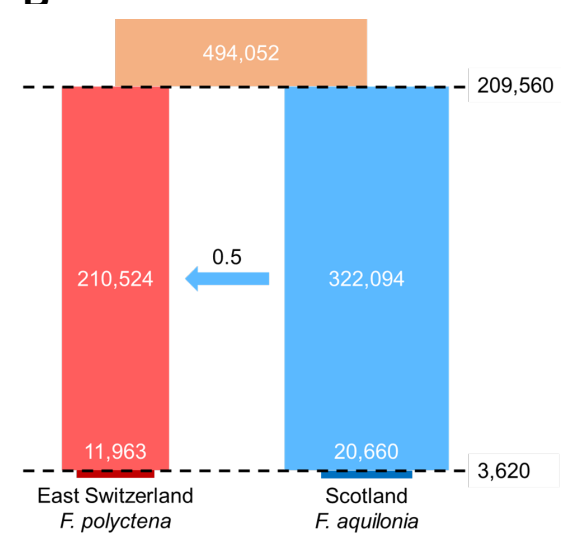

D

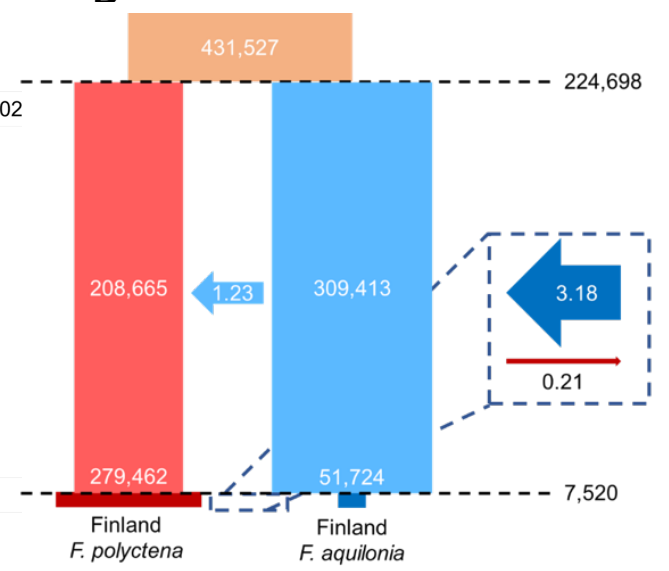

Figure 5 - Multiple sample pairs suggest a similar speciation history between Formica polyctena and $F$. aquilonia. Best divergence scenarios are depicted for the West Switzerland $F$. polyctena vs. Scotland $F$. aquilonia comparison $(\mathbf{A})$, the East Switzerland $F$. polyctena vs. Scotland $F$. aquilonia comparison (B), the West Switzerland $F$. polyctena vs. Switzerland $F$. aquilonia comparison (C) and the Finland $F$. polyctena vs. Finland $F$. aquilonia comparison (D). All times are given in number of generations and represented proportionally to each other across panels, as the time of divergence in panel A was taken as reference. All effective sizes are given in number of haploids. Sizes at a given time are represented proportionally to each other across panels, with the $F$. polyctena sizes in panel A serving as reference (i.e., all recent sizes are proportional to each other but not to ancestral sizes, while all ancestral sizes are proportional to each other but not to recent sizes). Arrows indicate the number of migrants per generation, their size is representative of this value. The direction and colour of the arrows are indicative of the direction of the gene flow. 


\section{Past gene flow between $F$. polyctena and $F$. aquilonia cannot be explained by migration from an unsampled sister species}

As several species from the F. rufa group are known to hybridize, we tested whether the patterns of gene flow inferred between $F$. polyctena and $F$. aquilonia could be caused by migration from an unsampled, "ghost" species. The expected likelihood of these models with "ghost" introgression was lower than all other "non-ghost" models, suggesting that gene flow between $F$. polyctena and $F$. aquilonia during divergence was the most parsimonious scenario for the samples considered in our study (Supplementary Tables 2 to 5 for the parameter estimates obtained with both models for all population pairs).

\section{DISCUSSION}

Divergence history between two related species is often inferred using samples from a single population pair assuming these samples capture the divergence history throughout the species' ranges. Yet, this assumption is rarely tested. In this study, we test this assumption by using whole-genome data from samples collected across the European distributions of Formica polyctena and $F$. aquilonia to reconstruct their speciation history. Using a moderate number of individuals from each geographic sampling site, we were able to infer consistent speciation histories across distinct sample pairs, both in terms of mode of divergence and parameter estimates (divergence times, ancestral population sizes). As we compared several different pairs of heterospecific populations, our approach also underlined how present-day species distributions affect the inference of the divergence history. Interestingly, we detected reduced gene flow at recent times between allopatric sampling locations while gene flow increased between sympatric Finnish populations in recent times. Finally, our coalescent simulations ruled out the alternative scenarios where gene flow inferred between $F$. polyctena and $F$. aquilonia would be actually caused by gene flow from a third, unsampled species. Below, we discuss the implications of our findings regarding divergence in the $F$. rufa group, and, more generally, the insights gained from contrasting two species across their ranges in terms of speciation research. 


\section{Samples from multiple locations yield a similar divergence history between $F$. polyctena and F. aquilonia}

All analyses carried out with several heterospecific samples across the species ranges supported the same scenario: $F$. polyctena and $F$. aquilonia diverged with gene flow. Formerly, the possibility of divergence in allopatry in different glacial refugia had been discussed for F. rufa group ants (Goropashnaya et al., 2004). However, it is not surprising that we found evidence for recent gene flow between $F$. polyctena and $F$. aquilonia, as many Formica species retain the ability to interbreed and produce viable offspring (Seifert and Goropashnaya, 2004, Kulmuni et al. 2010, Purcell et al., 2016) and interspecific gene flow has been previously described in many ant species (Feldhaar et al., 2008; Seifert, 2009; Steiner et al., 2011; Seifert, 2019). Yet, one of our most unexpected results is that gene flow during divergence is consistently inferred to be asymmetrical, with gene flow from $F$. aquilonia to $F$. polyctena. This would be observed if prezygotic isolation mechanisms were stronger in $F$. aquilonia than in $F$. polyctena or if $F$. aquilonia was more likely to disperse than $F$. polyctena. This signal of unidirectional gene flow could also be linked to a difficulty for $F$. polyctena individuals to find conspecific mates, which would be expected if this species has a smaller population size than $F$. aquilonia.

While the analyses of multiple heterospecific sample pairs supported the same divergence scenario, they also yielded similar divergence time estimates. The results obtained across all comparisons dated the divergence time between F. polyctena and F. aquilonia around 540,000 years on average, placing the divergence between both species in the Pleistocene. Our results are in line with previous estimates obtained using mitochondrial markers which dated the diversification of the $F$. rufa group (including F. polyctena and F. aquilonia) around 490,000 years ago (Goropashnaya et al. 2004, 2012). In all heterospecific population pairs considered, the effective size of the ancestral population of both $F$. polyctena and $F$. aquilonia was inferred between 460,000 and 500,000 haploid individuals. After the divergence of these species, $F$. aquilonia was always inferred to have a larger ancestral $N_{e}$ than $F$. polyctena and consistent $N_{e}$ estimates were obtained across models, indicating that samples of each 
species from different locations likely shared the same ancestral population. Due to the supercoloniality of both F. polyctena and F. aquilonia, we suggest that these species could follow the dynamics of a metapopulation (i.e. different supercolonies would be demes within a metapopulation). This could inflate our effective population size estimates, as coalescence of lineages within the same deme is expected to be faster than between lineages in different demes in a metapopulation (Wakeley, 2004). Under this scenario, lineages would be "trapped" within their demes before being able to coalesce with lineages from distinct demes. The maintenance of these lineages over longer time scales would therefore inflate the estimated effective population sizes. However, as both species in our study have similar colony structures, they should be equally impacted by this overestimation.

While the two study species are known to hybridize in Southern Finland (Kulmuni et al. 2010), they can also hybridize with other closely related species from the $F$. rufa group such as F. lugubris, F. paralugubris or F. rufa (Seifert and Goropashnaya, 2004; Seifert et al., 2010, Seifert 2021). In our ghost introgression models, we tested all possible scenarios of gene flow from an unsampled $F$. rufa group species into either $F$. polyctena or $F$. aquilonia, considering no direct gene flow between our two focal species. We did this to rule out the possibility that the signal of gene flow between our focal species is caused by gene flow from another, unsampled species. Under this hypothesis, the signal of gene flow from $F$. aquilonia into $F$. polyctena that we observe would be actually caused by gene flow from a sister species to $F$. aquilonia into $F$. polyctena. Our results indicate that such unaccounted migration from an unsampled species could not explain the observed pattern of migration between our two focal species. However, our approach relied on modeling unsampled lineages, where coalescent events might happen. In the future, these results should be confirmed by collecting the remaining species of the $F$. rufa group and reconstructing the speciation history of the group as a whole, allowing for gene flow from multiple lineages into the same focal species.

This work represents to our knowledge the first reconstruction of speciation histories between two ant species using genome-wide data. However, as for many non-model organisms, some key parameters required for inference remain unknown. The generation time we used was based on $F$. polyctena queen longevity estimates 
(Horstmann, 1983), while mutation rate was approximated using data from other social insects (honeybee and bumblebee mutation rates; reviewed in Liu et al., 2017). While these approximations should not bias our inferences, some uncertainty is associated with the date estimates provided throughout our manuscript.

\section{Present-day context of heterospecific sample pairs induces variability in inferred migration rates}

By comparing samples from multiple locations across both species ranges, our approach pinpointed commonalities in the divergence histories across all comparisons, providing a robust picture of the speciation history between $F$. polyctena and $F$. aquilonia. It also allowed us to document variation in the inferred estimates of certain parameters. As such, we can interpret this observed variation in parameter estimates in light of the present-day context of the heterospecific populations under consideration (i.e., whether or not they are presently in contact).

The Finnish F. polyctena population is clearly different where it comes to its recent $\mathrm{Ne}_{\mathrm{e}}$. Instead of estimates indicating a contraction at the time of size change, akin to the other populations of both $F$. polyctena and $F$. aquilonia, estimates suggest that this population has recently expanded. This seems unlikely, given that $F$. polyctena in Finland is at its range margins (Stockan \& Robinson 2016) and based on a national survey is the minority species in Finland (Punttila \& Kilpeläinen 2009). We suggest it is more likely that the signal of population expansion in Finnish F. polyctena is actually caused by gene flow.

Admixture between F. aquilonia and F. polyctena in Southern Finland is also supported by our results regarding population structure. Interspecific differentiation between the Finnish populations of $F$. polyctena and $F$. aquilonia was reduced and ancestry coefficients also suggested moderate introgression between both species in Finland. Finnish samples were also more genetically diverse than their conspecific populations sampled outside Finland. Particularly, the Finnish F. polyctena had the highest mean expected heterozygosity of all sampled populations, in line with the larger effective size inferred with our coalescent simulations. 
The bidirectional contemporary gene flow detected in Finnish populations could be expected under two non-mutually exclusive scenarios. The first is direct introgression of alleles from $F$. polyctena into $F$. aquilonia, which could be facilitated by human activity. The forest management strategy practiced in Finland results in the formation of sharp boundaries between habitats more suitable for $F$. aquilonia (old forests with shade and humidity) and areas where F. polyctena can establish, e.g. forest edges, as it can withstand increased exposure to sunlight (Punttila, 2020). This phenomenon might increase opportunities for direct contact between these two species due to closer proximity between heterospecific nests. The second scenario would involve the $F$. polyctena $\times$ F. aquilonia hybrid populations, which are common in Southern Finland (Beresford et al., 2017). These hybrid populations could mediate gene flow between the species via backcrosses between hybrids and individuals of the parental species (e.g., indirect gene flow). Elucidating these causes would require a dense survey of both parental and hybrid wood ant populations in Finland.

Variation in recent population effective sizes is to be expected as the current populations of these species would have evolved independently from each other, once the ancestral population of each species split into the ancestors of the sampled populations. Variation in recent $N_{\mathrm{e}}$ estimates obtained for the same population when compared to different heterospecific populations is likely caused by variation in the estimated time at which the size of the populations changes. When the time of size change is estimated to be older, the post-resize populations are estimated to have larger effective sizes.

\section{Implications for speciation research}

In this study, we used red wood ants to investigate how the divergence history between two species may vary depending on the geography of the pair of heterospecific populations considered. We sampled a relatively low number of individuals from each site but were able to infer consistent speciation histories across distinct sample pairs, both in terms of mode of divergence and parameter estimates. This suggests that speciation histories might be reliably reconstructed using a few individuals. However, the geographical context of the study populations is also of importance, as gene flow 
may be heterogeneous across both species ranges. This has implications for designing and/or interpreting studies aiming at reconstructing the divergence history between two or more taxa. The present-day context of the study populations should be taken into account when interpreting parameter estimates. More specifically, species-wide interpretations should be made cautiously when, e.g., only sympatric populations are used to perform demographic history reconstruction.

\section{CONCLUSIONS}

Here, we used genome-wide data to show that the mound-building wood ant species, $F$. polyctena and $F$. aquilonia, diverged with continuous asymmetrical gene flow across their ranges. Employing several heterospecific sample pairs and a coalescent-based inference method, we also demonstrated that the context in which present-day populations occur may influence the inference of certain demographic parameters, such as rates of migration between the populations, at different time scales. This work has important implications regarding the interpretation of studies carrying demographic history reconstruction. It will also pave the way to the study of admixture in hybrid wood ants, an emerging model in the genomics of hybridization.

\section{ACKNOWLEDGEMENTS}

We thank CSC - IT Center for Science, Finland, for computational resources. Our work was performed under the Global Ant Genomic Alliance and was supported by an HiLIFE fellowship and an Academy of Finland grant no. 309580 to JK. BP was funded through Societas pro Fauna et Flora Fennica and Erasmus+ grants. VCS was funded by "Fundação Ciência e Tecnologia" (Portuguese Science Foundation grants CEECINST/00032/2018/CP1523/CT0008 and CEECIND/02391/2017). PN thanks Dominik Laetsch for bioinformatic advice and Camille Lorry for assistance with fieldwork. The authors declare no conflicts of interest.

\section{AUTHOR CONTRIBUTIONS}

Study design: JK \& PN

Data collection: AA, CB, HH, JM, JK \& PN 
Morphological species identification: BS

Data analysis: BP \& PN

Data interpretation: BP, VCS, JK \& PN

Draft writing: BP, VCS, JK \& PN

Draft review and editing: all authors

Supervision: VCS, JK \& PN

Project funding: JK

\section{REFERENCES}

Beichman, A. C., Huerta-Sanchez, E., \& Lohmueller, K. E. (2018). Using genomic data to infer historic population dynamics of nonmodel organisms. Annu. Rev. Ecol. Evol. Syst., 49, 433-456.

Beresford, J., Elias, M., Pluckrose, L., Sundström, L., Butlin, R. K., Pamilo, P. et al. (2017). Widespread hybridization within mound-building wood ants in Southern Finland results in cytonuclear mismatches and potential for sex-specific hybrid breakdown. Mol. Ecol., 26(15), 4013-4026.

Bolger, A. M., Lohse, M., \& Usadel, B. (2014). Trimmomatic: A flexible trimmer for Illumina sequence data. Bioinformatics, 30(15), 2114-2120.

Boomsma, J. J., Brady, S. G., Dunn, R. R., Gadau, J., Heinze, J., Keller, L. et al. (2017). The Global Ant Genomics Alliance (GAGA). Myrmecol. News, 25(October), 61-66.

Bradburd, G. S. \& Ralph, P. L. (2019). Spatial population genetics: it's about time. ArXiv.

Brelsford, A., Purcell, J., Avril, A., Tran Van, P., Zhang, J., Brütsch, T. et al. (2020). An Ancient and Eroded Social Supergene Is Widespread across Formica Ants. Curr. Biol., 30(2), 304-311.e4.

Cherix, D., Bernasconi, C., Maeder, A., \& Freitag, A. (2012). Fourmis des bois en suisse: état de la situation et perspectives de monitoring. Schweiz. Z. Forstwes., 163(6), 232-239.

Currat, M., Ruedi, M., Petit, R. J., \& Excoffier, L. (2008). The hidden side of invasions: Massive introgression by local genes. Evolution, 62(8), 1908-1920.

De La Filia, A. G., Bain, S. A., \& Ross, L. (2015). Haplodiploidy and the reproductive ecology of Arthropods. Curr. Opin. Insect Sci., 9, 36-43.

Excoffier, L., Dupanloup, I., Huerta-Sánchez, E., Sousa, V. C., \& Foll, M. (2013). Robust Demographic Inference from Genomic and SNP Data. PLoS Genet., 9(10).

Feldhaar, H., Foitzik, S., \& Heinze, J. (2008). Lifelong commitment to the wrong partner: Hybridization in ants. Philos. Trans. R. Soc. B Biol. Sci., 363(1505), 2891-2899.

Filatov, D. A., Osborne, O. G., \& Papadopulos, A. S. T. (2016). Demographic history of speciation in a Senecio altitudinal hybrid zone on Mt. Etna. Mol. E., 25(11), 2467-2481.

Frichot, E., \& François, O. (2015). LEA: An R package for landscape and ecological association studies. Methods Ecol. Evol., 6(8), 925-929.

Frichot, E., Mathieu, F., Trouillon, T., Bouchard, G., \& François, O. (2014). Fast and efficient estimation of individual ancestry coefficients. Genetics, 196(4), 973-983.

Frouz J, Jílková V \& Sorvari J. (2016). Contribution of wood ants to nutrient cycling and ecosystem function. In: Wood Ant Ecology and Conservation. Cambridge University Press pp. 207-220.

Garcia-Erill, G., Kjær, M. M., Albrechtsen, A., Siegismund, H. R., \& Heller, R. (2021). Vicariance followed by secondary gene flow in a young gazelle species complex. Mol. Ecol., 30(2), 528-544.

Garrison, E., \& Marth, G. (2012). Haplotype-based variant detection from short-read sequencing. aRxiv

Goropashnaya, A. V., Fedorov, V. B., \& Pamilo, P. (2004). Recent speciation in the Formica rufa group ants (Hymenoptera, Formicidae): Inference from mitochondrial DNA phylogeny. Mol. Phylogenet. Evol., 32(1), 198-206. 
Goropashnaya, A. V., Fedorov, V. B., Seifert, B., \& Pamilo, P. (2012). Phylogenetic relationships of Palaearctic Formica species (Hymenoptera, Formicidae) based on mitochondrial cytochrome b sequences. PLOS ONE, 7(7).

Green, R. E., Krause J., Briggs A. W., Maricic T., Stenzel U., et al. (2010). A Draft Sequence of the Neandertal Genome. Science 328(5979), 710-22.

Ito, T., Kanthaswamy, S., Bunlungsup, S., Oldt, R. F., Houghton, P., Hamada, Y. et al. (2020). Secondary contact and genomic admixture between rhesus and long-tailed macaques in the Indochina Peninsula. J. Evol. Biol., 33(9), 1164-1179.

Kulmuni, J., Seifert, B., \& Pamilo, P. (2010). Segregation distortion causes large-scale differences between male and female genomes in hybrid ants. Proc. Natl. Acad. Sci. U. S. A., 107(16), 73717376.

Li, H., \& Durbin, R. (2010). Fast and accurate long-read alignment with Burrows-Wheeler transform. Bioinformatics, 26(5), 589-595.

Maeder, A. et al. (2016). Wood ant reproductive biology and social systems. In: Wood Ant Ecology and Conservation. Cambridge University Press pp. 37-50.

Nouhaud, P., Beresford, J. \& Kulmuni, J. (2021). Cost-effective long-read assembly of a hybrid Formica aquilonia $\times$ Formica polyctena wood ant genome from a single haploid individual. bioRxiv 2021.03.09.434597

Pabijan, M., Zieliński, P., Dudek, K., Stuglik, M., \& Babik, W. (2017). Isolation and gene flow in a speciation continuum in newts. Mol. Phylogenet. Evol., 116(August), 1-12.

Pedersen, B. S., \& Quinlan, A. R. (2018). Mosdepth: Quick coverage calculation for genomes and exomes. Bioinformatics, 34(5), 867-868.

Punttila, P. (2020). Ant community structure in successional mosaics of boreal forests.

Punttila, P., \& Kilpelainen, J. (2009). Distribution of mound-building ant species (Formica spp., Hymenoptera) in Finland: preliminary results of a national survey. Ann. Zool. Fennici, 46(1), 1-15.

Purcell, J., Zahnd, S., Athanasiades, A., Türler, R., Chapuisat, M., \& Brelsford, A. (2016). Ants exhibit asymmetric hybridization in a mosaic hybrid zone. Mol. Ecol., 25(19), 4866-4874.

Pfeifer, S. P., Laurent, S., Sousa, V. C., Linnen, C. R., Foll, M., Excoffier, L. et al. (2018). The evolutionary history of Nebraska deer mice: Local adaptation in the face of strong gene flow. Mol. Bio. Evol., 35(4), 792-806.

R Core Team (2017). R: A Language and Environment for Statistical Computing. https://www.Rproject.org/

Ravinet, M., Faria, R., Butlin, R. K., Galindo, J., Bierne, N., Rafajlović, M. et al. (2017). Interpreting the genomic landscape of speciation: a road map for finding barriers to gene flow. J. Evol. Biol., 30(8), 1450-1477.

Rougemont, Q., \& Bernatchez, L. (2018). The demographic history of Atlantic salmon (Salmo salar) across its distribution range reconstructed from approximate Bayesian computations. Evolution, 72(6), 1261-1277.

Seifert, B. (2009). Cryptic species in ants (Hymenoptera: Formicidae) revisited: we need a change in the alpha-taxonomic approach. Myrmecol. News, 12(June), 149-166.

Seifert, B. (2018). The ants of Central and North Europe:. Boxberg: Lutra, 407 pp.

Seifert, B. (2019). Hybridization in the European carpenter ants Camponotus herculeanus and $C$. ligniperda (Hymenoptera: Formicidae). Insectes Soc., 66(3), 365-374.

Seifert, B. (2021). A taxonomic revision of the Palaearctic members of the Formica rufa group (Hymenoptera, Formicidae) - the famous mound-building red wood ants. Myrmecol. News, in press.

Seifert, B., \& Goropashnaya, A. V. (2004). Ideal phenotypes and mismatching haplotypes - Errors of mtDNA treeing in ants (Hymenoptera: Formicidae) detected by standardized morphometry. Org. Divers. Evol., 4(4), 295-305. 
Seifert, B., Kulmuni, J., \& Pamilo, P. (2010). Independent hybrid populations of Formica polyctena X rufa wood ants (Hymenoptera: Formicidae) abound under conditions of forest fragmentation. Evol. Ecol., 24(5), 1219-1237.

Sousa, V. \& Hey, J. (2013). Understanding the origin of species with genome-scale data: Modelling gene flow. Nat. Rev. Genet., 14(6), 404-414.

Steiner, F. M., Seifert, B., Grasso, D. A., Le Moli, F., Arthofer, W., Stauffer, C. et al. (2011). Mixed colonies and hybridisation of Messor harvester ant species (Hymenoptera: Formicidae). Org. Divers. Evol., 11(2), 107-134.

Stockan, J.A. et al. (2016). Introducing wood ants: evolution, phylogeny, identification and distribution. In: Wood Ant Ecology and Conservation. Cambridge University Press pp. 1-36.

Sundström, L., Seppä, P., \& Pamilo, P. (2005). Genetic population structure and dispersal patterns in Formica ants - A review. Ann. Zool.Fennici, 42(3), 163-177.

Sutra, N., Kusumi, J., Montenegro, J., Kobayashi, H., Fujimoto, S., Masengi, K. W. A. et al. (2019). Evidence for sympatric speciation in a Wallacean ancient lake. Evolution, 73(9), 1898-1915.

Tan, A., Abecasis, G. R., \& Kang, H. M. (2015). Unified representation of genetic variants. Bioinformatics, 31(13), 2202-2204.

Wakeley, J. (2004). Metapopulation models for historical inference. Mol. Ecol., 13(4), 865-875.

Weir, B. S., \& Cockerham, C. C. (1984). Estimating F-Statistics for the Analysis of Population Structure. Evolution, 38(6), 1358.

Yagi, H., Xu, J., Moriguchi, N., Miyagi, R., Moritsuka, E., Sato, E. et al. (2019). Population genetic analysis of two species of Distylium: $D$. racemosum growing in East Asian evergreen broad-leaved forests and $D$. lepidotum endemic to the Ogasawara (Bonin) Islands. Tree Genet. Genomes, 15(6).

Zieliński, P., Nadachowska-Brzyska, K., Dudek, K., \& Babik, W. (2016). Divergence history of the Carpathian and smooth newts modelled in space and time. Mol. Ecol., 25(16), 3912-3928.

Zheng, X., Levine, D., Shen, J., Gogarten, S. M., Laurie, C., \& Weir, B. S. (2012). A high-performance computing toolset for relatedness and principal component analysis of SNP data. Bioinformatics, 28(24), 3326-3328. 